\title{
Faut-il éradiquer les Loranthaceae sur les ligneux à fruits commercialisés de la région littorale du Cameroun?
}

\author{
Siegfried Didier DIBONG ${ }^{1 *}$, Bruno NGOTTA BIYON ${ }^{1}$, Nestor Laurier ENGONE \\ $\mathrm{OBIANG}^{2}$, Ndongo DIN ${ }^{1}$, R. Jules PRISO ${ }^{1}$, Victor Désiré TAFFOUO, Henri \\ FANKEM $^{1}$, Georges SALLE ${ }^{3}$, Alain Didier MISSOUP ${ }^{4}$, Joseph ISSAKA \\ BOUSSIM $^{5}$ et AMOUGOU AKOA ${ }^{6}$ \\ ${ }^{I}$ Laboratoire d'Ecologie Végétale, Département de Biologie des Organismes végétaux, Faculté des Sciences, \\ B. P. 24157, Université de Douala, Cameroun. \\ ${ }^{2}$ Institut de Recherche d'Ecologie Tropicale (IRET), B. P. 13354 Libreville, Gabon. \\ ${ }^{3}$ Laboratoire de Parasitologie végétale, Université Pierre et Marie Curie (Paris VI), 4 place Jussieu, case \\ courrier 155, 75252 Paris Cedex 05, France. \\ ${ }^{4}$ Département de Biologie des Organismes Animaux, Faculté des Sciences, B. P. 24157, Université de Douala, \\ Cameroun; UMR 7205, MNHN, Paris, 55 Rue Buffon, France. \\ ${ }^{5}$ Laboratoire de Biologie et d'Ecologie Végétales, Université de Ouagadougou 03 B. P. 848, Ouagadougou \\ 03, Burkina Faso. \\ ${ }^{6}$ Département de Biologie et Physiologie Végétales, B. P. 812, Université de Yaoundé I, Cameroun. \\ *Auteur correspondant, E-mail: didierdibong @yahoo.fr
}

\section{RESUME}

Dans la région du littoral camerounais, Phragmanthera capitata (généraliste) et Tapinanthus ogowensis (spécialiste) sont majoritaires dans la plupart des agroécosystèmes. Les deux Loranthaceae attaquent les essences cultivées ou spontanées d'importance économique ou non, réduisent les rendements et détériorent la qualité des fruits sous l'effet néfaste et conjugué de la salinité des sols. Les hémiparasites prélèvent sur leurs hôtes de l'eau et des substances minérales nécessaires à leur développement. Le comportement généraliste ou spécialiste des deux Loranthaceae sur la réduction des concentrations en $\mathrm{Na}+$ et $\mathrm{K}+$ a été étudié sur cinq ligneux parasités. Le dosage du $\mathrm{Na}+$ et $\mathrm{du} \mathrm{K}+$ par spectrophotométrie de flamme sur 30 échantillons appartenant à cinq couples Loranthaceae/hôte ont révélé que les concentrations moyennes de $\mathrm{Na}+$ varient entre $5,76 \pm 0,68$ et $33,74 \pm 1,31 \mathrm{~g} / \mathrm{kg}$ de $\mathrm{ms}$ chez les hôtes et entre 8,66 $\pm 1,84$ et $19,84 \pm 1,26 \mathrm{~g} / \mathrm{kg}$ de ms chez les Loranthaceae. Les concentrations moyennes de $\mathrm{K}+$ varient entre $16,32 \pm 1,54$ et $143,08 \pm 2,01 \mathrm{~g} / \mathrm{kg}$ de ms chez les hôtes et entre $36,18 \pm 2,86$ et $122,32 \pm 1,72 \mathrm{~g} / \mathrm{kg}$ de $\mathrm{ms}$ chez les Loranthaceae. Les minima chez les hôtes correspondent à la racine et chez les Loranthaceae au suçoir. Les maxima chez les hôtes correspondent aux feuilles des branches non parasitées et chez les Loranthaceae aux feuilles. Ces résultats discutés en fonction de la biologie des hémiparasites suggèrent que l'éradication n'est pas nécessaire compte tenu de leur atout dans la pharmacopée traditionnelle. Toutefois la compréhension des interactions parasitaires des acteurs en présence permettrait le contrôle des Loranthaceae et la préservation de la biodiversité.

(C) 2010 International Formulae Group. All rights reserved.

Mots clés: Ligneux, salinité, Loranthaceae, rendement, lutte, littoral Camerounais

\section{INTRODUCTION}

En Afrique tropicale et particulièrement au Cameroun, les Loranthaceae sont très communes sur les ligneux d'intérêt économique ou non. Ce sont les phanérogames épiphytoïdes hémiparasites qui 
forment des touffes plus ou moins sphériques sur les parties aériennes de l'hôte (Dibong et al., 2008 ; Dibong et al., 2009f). Capables d'effectuer la photosynthèse et donc d'élaborer les substances nécessaires à leur nutrition carbonée, ils se caractérisent par la mise en place dans les tissus de l'hôte, d'un appareil d'absorption particulier appelé suçoir ou haustorium. Ce dernier est un véritable pont structural et physiologique régulant plus ou moins finement les débits de sève brute par le biais des connexions xylémiques (Boussim, 2002). Extérieurement, la zone de fixation du parasite sur l'hôte montre une réaction de ce dernier au parasitisme. Celle-ci forme une boursouflure plus ou moins volumineuse selon sa nature et l'espèce de Loranthaceae considérée. En coupe longitudinale axiale, le suçoir en forme de « $\mathrm{V} »$, est profondément enfoncé dans le bois de l'hôte.

Le suçoir assure une continuité des éléments conducteurs des deux plantes et permet le détournement de l'eau et des substances nutritives de l'hôte vers le parasite (Bannister et al., 2002). Cette spoliation trophique au profit du parasite entraîne une sous-alimentation de la partie de la branche située en aval de la zone de fixation du parasite qui se traduit par un diamètre faible. Cette partie de la branche peut ensuite se dessécher. Finalement, elle peut tomber, amenant ainsi secondairement la touffe de Loranthaceae en position terminale.

La famille des Loranthaceae, largement répandue dans le monde, comprend 77 genres et 950 espèces (Polhill et Wiens, 1998). Sept genres et une trentaine d'espèces sont signalés au Cameroun (Balle, 1982). Parmi ces espèces, Phragmanthera capitata (Sprengel) Balle et Tapinanthus ogowensis (Engler) Danser sont majoritaires dans la région littorale (Dibong et al., 2009a) et attaquent la plupart des arbres cultivés (agrumes, avocatiers, cacaoyers, caféiers, goyaviers...) ou spontanés dont les fruits sont commercialisés et exportés. Il en résulte un détournement trophique de l'eau et des éléments minéraux, responsable d'importantes pertes économiques (Sonké et al., 2000). Dans la région littorale, le taux de salinité élevé des sols littoraux accentue cette baisse de rendement et entame la qualité des fruits notamment les agrumes. Les fruits au goût très salé deviennent impropres à la consommation. Les éléments minéraux impliqués dans cette salinité sont les ions $\mathrm{Na}+$ L'hypothèse formulée est que les Loranthaceae contribueraient à diminuer la teneur en sel sur les branches hôtes parasitées et donc sur les rameaux fructifères.

Le présent travail consiste à vérifier cette hypothèse à partir de la répartition du $\mathrm{K}+$ et $\mathrm{Na}+$ sur Phragmanthera capitata (généraliste) et Tapinanthus ogowensis (spécialiste) et leur (s) hôte (s).

\section{MATERIEL ET METHODES}

\section{Le site d'étude}

Douala (latitude, $03^{\circ} 40-04^{\circ} 11^{\prime} \mathrm{N}$; longitude, $09^{\circ} 16^{\prime}-09^{\circ} 52^{\prime} \mathrm{E}$; altitude, $13 \mathrm{~m}$ ) a un climat qui appartient au domaine équatorial du type «camerounien » qui se caractérise par deux saisons avec une longue saison de pluies (au moins 9 mois), des précipitations abondantes (environ $4000 \mathrm{~mm}$ par an), des températures élevées $\left(26,7{ }^{\circ} \mathrm{C}\right)$ et stables. La moyenne minimale de température à Douala pour 30 années (1961-1990) est de 22,6 ${ }^{\circ} \mathrm{C}$ en juillet et la moyenne maximale de température de $32,3{ }^{\circ} \mathrm{C}$ en février. L'humidité relative de l'air reste élevée toute l'année et voisine de $100 \%$ (Din et al., 2008).

\section{Matériel végétal}

Deux Loranthaceae et cinq hôtes dont quatre parasités par Phragmanthera capitata et le cinquième Dacryodes edulis parasité Tapinanthus ogowensis ont été utilisés. La première Loranthaceae est généraliste et la seconde spécialiste dans les agroécosystèmes du littoral camerounais (Dibong et al., 2009f). Les hôtes parasités par $P$. capitata sont: Citrus maxima, Persea americana, Psidium guajava et Theobroma cacao. 
Méthodes d'étude

Sur le terrain

Les échantillons ont été récoltés au verger du campement de la chefferie de Ndogbong, quartier situé au nord-est de la ville de Douala. Le verger a une superficie de 8 ha environs et constitué essentiellement d'arbres fruitiers dont les fruits sont comestibles.

\section{Choix des espèces hôtes et parasites}

Les espèces hôtes retenues sont des arbres fruitiers cultivés ou spontanés. Les fruits commercialisés sont très appréciés des consommateurs et procurent des revenus importants. Les deux parasites appartiennent à la famille des Loranthaceae, l'un est généraliste et l'autre spécialiste suggérant un comportement différent dans la distribution du $\mathrm{Na}+$ et du K+ avec leur (s) hôte (s).

Les prélèvements ont été faits en juillet 2009 sur 5 individus hôtes par couple soit au total 30 échantillons. Pour chaque couple, les racines de l'hôte $(\mathrm{RH})$, les suçoirs du parasite $(\mathrm{SPa})$, les feuilles du parasite $(\mathrm{FPa})$, les feuilles des branches de l'hôte non parasitées (FNPH) et les feuilles de la partie distale des branches parasitées (FPH) ont été prélevés. Le stockage et le transport au laboratoire se sont faits dans des sacs en plastique de couleur noire.

\section{Au laboratoire}

Le séchage à l'étuve s'est fait immédiatement après le transport au laboratoire de Biologie des Organismes Végétaux de la Faculté des Sciences de l'Université de Douala. Les échantillons ont été placés à la température de $60{ }^{\circ} \mathrm{C}$ pendant 96 heures avant d'être broyés au moulinex et conservés à la température ambiante jusqu'au moment du dosage.

Après minéralisation de chaque partie du parasite et de l'hôte à analyser, le $\mathrm{K}+$ et le $\mathrm{Na}+$ ont été dosés par spectrophotomètre de flamme (JENWAY).

\section{Analyse statistique}

Les résultats sont donnés en terme de moyenne \pm déviation standard. Les analyses statistiques sont réalisées à l'aide du logiciel StatSoft, Inc. (2001) STATISTICA (data analysis software system) version 6.0. La comparaison simultanée des moyennes est réalisée au test de l'ANOVA et les tests analytiques entre le lot témoin et les lots expérimentaux sont effectués suivant la procédure de Dunnett lorsque les conditions de normalité et d'égalité des variances sont vérifiées. $\mathrm{P}<0.05 ; \mathrm{P}<0.01$ and $\mathrm{p}<0.001$.

\section{RESULTATS}

Impact économique des Loranthaceae

Selon les paysans de la région littorale interrogés, la lutte mécanique contre le parasitisme des Loranthaceae expose très souvent les fruits des arbres hôtes aux maladies fongiques. La qualité des fruits est entamée et les baisses de rendement importantes. La désolation est si grande que l'autosuffisance jadis brandie comme un slogan politique est compromise. Le même constat a été fait également in situ dans les jardins de cases et les vergers du site étudié. Le parasitisme de l'avocatier est plus perceptible. En effet, cet arbre fruitier fréquent et abondant dans les agroécosystèmes de la région est sensible au parasitisme des Loranthaceae. Les fruits contiennent des crevasses et des pustules et sont impropres à la consommation.

La pharmacopée traditionnelle dans la médication pour le traitement des maladies est très pratiquée par la majorité des populations rurales de la région littorale. En effet, cette pharmacopée reste à la portée de toutes les bourses. Les Loranthaceae sont reconnues par les tradipraticiens de cette région comme possédant de nombreuses vertus thérapeutiques.

\section{Répartition du $\mathrm{K}+$ et du $\mathrm{Na}+$ entre les parasites vasculaires et leur (s) hôte (s)}

Les concentrations moyennes de $\mathrm{Na}+$ varient entre $5,76 \pm 0,68$ et $33,74 \pm 1,31 \mathrm{~g} / \mathrm{kg}$ de $\mathrm{ms}$ chez les hôtes; entre 8,66 $\pm 1,64$ et $19,84 \pm 1,26 \mathrm{~g} / \mathrm{kg}$ de $\mathrm{ms}$ chez les Loranthaceae (Figure 1 et 2). Les concentrations de $\mathrm{K}+$ varient entre 16,32 \pm 1,54 et $143,08 \pm 2,01 \mathrm{~g} / \mathrm{kg}$ de $\mathrm{ms}$ chez les hôtes; entre $36,18 \pm 2,86$ et $199,78 \pm 2,44$ $\mathrm{g} / \mathrm{kg}$ de ms chez les Loranthaceae (Figure 1 et 2). Les minima chez les hôtes correspondent aux racines et chez les Loranthaceae, aux suçoirs. Les maxima chez les hôtes correspondent aux feuilles des branches non 
parasitées et chez les Loranthaceae, aux feuilles (Figures 1 et 2).

Les tests ANOVA et LSD des variances des concentrations moyennes en $\mathrm{Na}+$ et $\mathrm{K}+$ du suçoir et des feuilles des Loranthaceae d'une part et d'autre part des feuilles des branches parasitées ou non des hôtes ainsi que des racines des hôtes et des suçoirs des Loranthaceae donnent des valeurs hautement significatives $(\mathrm{p}<0,001)$.
Les concentrations moyennes en $\mathrm{Na}+$ et $\mathrm{K}+$ des feuilles de Loranthaceae sont toujours plus élevées que celles des feuilles parasitées des hôtes. Les concentrations moyennes en $\mathrm{Na}+$ des parties des branches non parasitées des hôtes sont toujours plus élevées que celles des parties équivalentes parasitées. Il en est de même des concentrations moyennes en $\mathrm{K}+$ (Figures 1 et 2).
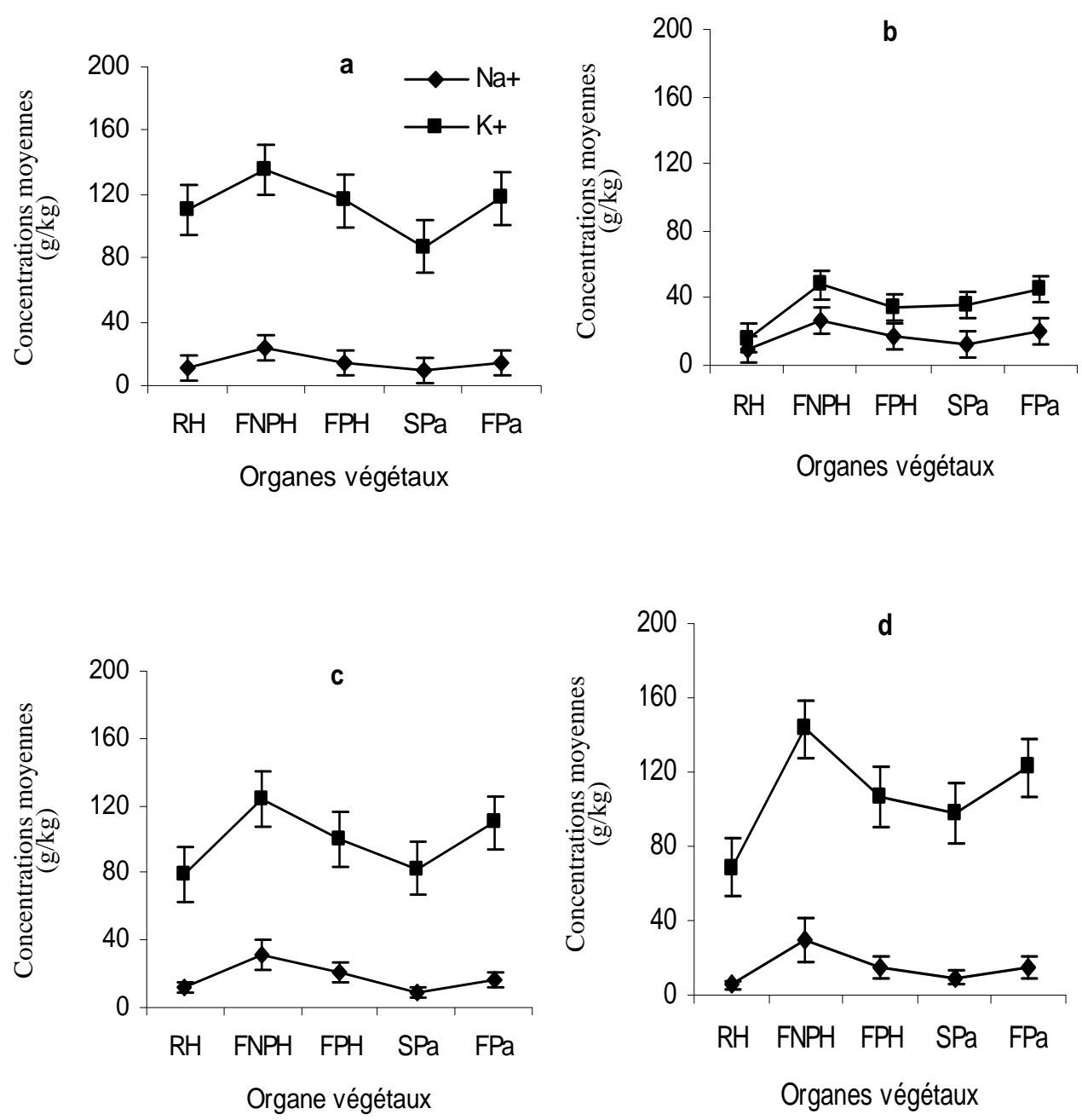

Figure 1 : Concentrations en $\mathrm{Na}^{+}$et $\mathrm{K}^{+}$des ligneux parasités par une Loranthaceae généraliste (Phragmanthera capitata) dans la région de Douala.

RH : racines de l'hôte ; FNPH : feuilles non parasitées de l'hôte ; FPH : feuilles parasitées de l'hôte ; SPa : suçoir du parasite ; FPa : feuilles du parasite. a : Citrus maxima, b : Persea Americana, c: Psidium guajava, d: Theobroma cacao; les barres verticales représentent la déviation standard. 


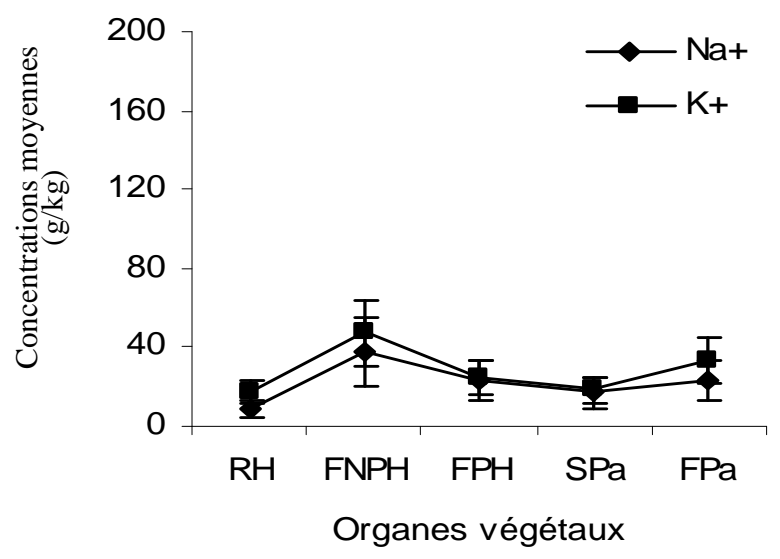

Figure 2 : Concentrations en $\mathrm{Na}^{+}$et $\mathrm{K}^{+}$des ligneux parasités par une Loranthaceae spécialiste (Tapinanthus ogowensis) dans la région de Douala.

RH : racines de l'hôte ; FNPH : feuilles non parasitées de l'hôte ; FPH : feuilles parasitées de l'hôte ; SPa : suçoir du parasite ; FPa : feuilles du parasite. Les barres verticales représentent la déviation standard.

\section{DISCUSSION}

Une caractéristique physiologique saillante de la plupart des plantes parasites est le degré élevé de la transpiration qui souvent excède celui de l'hôte (Glatzel, 1983). Il se crée un gradient de potentiel hydrique sur les feuilles du parasite qui facilite le flux des ressources nutritives. Ce mécanisme est commun chez les parasites épiphytes des régions tropicales. Le degré de transpiration des plantes parasites est le plus élevé parmi les Angiospermes (Whittington et Sinclair, 1988).

Les pertes d'eau par transpiration, élevées chez les Loranthaceae sont sous contrôle stomatique. Il en résulte un gradient hydrostatique qui facilite le transfert de solutés de l'hôte vers le parasite.

Les espèces hôtes accumulent le $\mathrm{Na}+$ dans les racines et les parties aériennes. Elles sont dites glycophytes sensibles. Selon Levitt (1980), le seuil de tolérance varie de 0 à 50 $\mathrm{mM}$ de $\mathrm{NaCl}$. L'effet suppressif du sel sur la croissance de la plante s'exprime davantage sur les parties aériennes que sur les racines des espèces hôtes. Les minima de $\mathrm{Na}+$ et $\mathrm{K}+$ observés concernent les racines des hôtes et les suçoirs des Loranthaceae: ce sont des organes de transitent. Les suçoirs des Loranthaceae joueraient le même rôle que les racines des hôtes. Ces deux organes absorbent les éléments minéraux sous forme d'ions et les distribuent dans le reste de la plante, notamment les feuilles.

Les maxima de $\mathrm{Na}+$ et $\mathrm{K}+$ observés concernent les feuilles non parasitées des branches des hôtes et celles des Loranthaceae : ce sont des organes d'accumulation. Toutefois, pour un même type de feuille, les concentrations en $\mathrm{Na}+$ sont toujours inférieures aux concentrations en $\mathrm{K}+$. La région littorale est située sur la zone côtière atlantique et par conséquent constituée de sols salins (Taffouo, 2005). La sécheresse, accentuée par les changements climatiques, accroît la salinité des sols et induit de fortes pressions osmotiques qui limitent l'absorption de l'eau par les cultures (Munns, 2002). Ainsi, la concentration saline du sol constitue le principal facteur responsable de la dégradation des sols mais aussi de la baisse du rendement des produits agricoles et de leur qualité. La spécificité de l'hôte aux parasites serait favorisée par les avantages d'adaptation aux interactions avec un hôte fréquemment rencontré (Norton et Carpenter, 1998). Le 
statut de généraliste ( $P$. capitata) serait avantageux, spécialement dans une communauté hétérogène en ce sens que celleci permet au parasite de croître avec succès sur plusieurs hôtes potentiels rencontrés. Quand les populations hôtes sont incertaines et éphémères, les généralistes sont plus vraisemblablement sollicités (Thompson, 1994). L'abondance relative des espèces hôtes est alors un facteur déterminant le degré de spécificité de l'hôte au parasite (Norton et Carpenter, 1998). Le degré de spécificité de l'hôte serait influencé également par le temps pendant lequel le parasite et son hôte ont été associés. La seconde règle de Mantel stipule qu'une longue association entre le parasite et son hôte aboutira à une spécificité élevée (Brook et Mc Lennan, 1993). Shaw (1994) pense que la spécificité des espèces hôtes serait étroitement associée aux groupements paucispécifiques. Tel est le cas de Tapinanthus ogowensis qui ne parasite que Dacryodes edulis dans la région littorale (Dibong et al., 2009a). Pourtant au Gabon, cette Loranthaceae parasite plusieurs espèces hôtes parmi lesquelles Casuarina equisetiformis (filao), Citrus deliciosa (mandarinier), C. limon (citronnier), $C$. maxima (pamplemoussier), $C$. sinensis (oranger), Dacryodes edulis (safoutier), Hevea brasiliensis (hévéa), Theobroma cacao (cacaoyer).

Dans la région littorale, huit Loranthaceae ont été recensées et Phragmanthera capitata (généraliste) est la plus fréquente et la plus abondante aussi bien dans les groupements végétaux homogènes (plantations d'hévéa) que dans les groupements hétérogènes (agroécosystèmes) (Dibong et al., 2009b). Deux méthodes de lutte ont été couramment pratiquées, l'élagage et la lutte chimique à l'éthéphon (Dibong et al., 2010). Mais celles-ci ont montré leurs limites. Dans le cas de la lutte mécanique, certains paysans opèrent la suppression des touffes du parasite sur le suçoir provoquant une régénération rapide des nouvelles branches de ce dernier après quelques semaines (Dibong et al., 2009e; Dibong et al.,
2010). L'usage de l'éthéphon contre les Loranthaceae (Coder, 2003) n'a aucun effet sur $P$. capitata puisque, bien que l'encoche soit régulièrement badigeonnée pour stimuler la production, le parasite est toujours présent (Engone et Sallé, 2006).

\section{Conclusion}

Les Loranthaceae jouent un rôle important dans le littoral camerounais en réduisant la salinité des arbres hôtes et en améliorant la saveur des fruits notamment des agrumes. Cette famille constitue un atout pour l'essor de la pharmacopée traditionnelle au Cameroun (Dibong et al., 2009c, 2009d). Cependant, les pertes de rendement doivent être chiffrées pour connaître leur impact sur l'économie. Des travaux menés au Gabon par Engone et Sallé (2006) concernant l'effet de $P$. capitata sur la production en caoutchouc de trois clones d'hévéa ont montré que l'éradication de cette Loranthaceae n'a pas lieu d'être proposée pour le moment. Toutefois, pour la maîtrise des interactions entre les différents acteurs en jeu (Mony et al., 2009, 2010), il serait nécessaire de promouvoir l'agroécologie afin de contrôler les parasites et de préserver la biodiversité des agroécosystèmes.

\section{BIBLIOGRAPHIE}

Balle S. 1982. Loranthacées, Flore du Cameroun (vol. 23), Satabié B, Leroy JF (eds). Yaoundé, Cameroun ; p. 82.

Bannister P, Graham L, Strong, Inge A. 2002. Is Differential accumulation of elements leaves of Mistletoes and their hosts related to greater water loss in Mistletoes? 3rd Int. Canopy Conf., Cairns, Australia.

Boussim IJ. 2002. Les phanérogames parasites du Burkina Faso : inventaire, taxonomie, écologie et quelques aspects de leur biologie. Cas particulier des Loranthaceae parasites du karité. Thèse de Doctorat d'Etat, Université de Ouagadougou, p. 285.

Brooks DR, Mc Lennan DA. 1993. Parasites and the Language of Evolution. 
Smithsonian Institution Press: Washington and London.

Coder KD. 2003. Treating Mistletoe (Phoradendron serotinum) in trees. University of Georgia School of Forest Resource, publication FOR 3-10, p. 2.

Dibong SD, Din N, Priso RJ, Taffouo VD, Fankem Henri, Sallé G, Amougou A. 2008. Parasitism of host trees by the Loranthaceae in the region of Douala (Cameroon). African Journal of Environmental Science and Technology, 2(11): 371-378.

Dibong SD, Engoné Obiang NL, Din N, Priso RJ, Taffouo VD, Fankem Henri, Sallé G, Amougou A. 2009a. Artificial infestations of Tapinanthus ogowensis (Engler) Danser (Loranthaceae) on three host species in the Logbessou plateau (Douala, Cameroon). African Journal of Biotechnology, 8(6): 1044-1051.

Dibong SD, Engoné Obiang NL, Din N, Priso RJ, Taffouo VD, Fankem Henri, Sallé G, Amougou A. 2009b. Niveau d'infestation des arbres fruitiers des groupements végétaux par Phragmanthera capitata (Sprengel) Balle (Loranthaceae) dans la région littorale du Cameroun. International Journal of Biological and Chemical Sciences, 3(2): 347-354.

Dibong SD, Engoné Obiang NL, Din N, Priso RJ, Taffouo VD, Fankem Henri, Sallé G, Missoup AD, Boussim IJ, Amougou A. 2009c. An assessment on the uses of Loranthaceae in ethnopharmacology in Cameroon: a case study made in Logbessou, north of Douala. Journal of Medicinal Plants Research, 3(8): 592595.

Dibong SD, Engoné Obiang NL, Din N, Priso RJ, Taffouo VD, Fankem Henri, Sallé G, Amougou A. 2009d. Les Loranthaceae: un atout pour l'essor de la pharmacopée traditionnelle au Cameroun. International Journal of Biological Chemical Sciences, 3(4): 746-754.

Dibong SD, Din N, Priso RJ, Taffouo VD, Sallé G, Amougou A. 2009e.
Germination et régénération naturelle de Phragmanthera capitata (Loranthaceae) sur les arbres fruitiers à Douala, Cameroun. In Systématique et Conservation des Plantes Africaines, X Van der Burgt, J van der Maesen, JM Onana (eds). Royal Botanic Gardens: Kew; 839-846.

Dibong SD, Din N, Priso RJ, Taffouo VD, Sallé G, Amougou A. 2009f. Statut écologique des Loranthaceae de la region littorale du Cameroun. In Systématique et Conservation des Plantes Africaines, X Van der Burgt, J van der Maesen, JM Onana (eds). Royal Botanic Gardens: Kew; 797-803.

Dibong SD, Mony R, Ndiang Zenabou, Ondoua JM, Boussim IJ, Bilong Bilong, Amougou A. 2010. Which struggle against Phragmanthera capitata (Sprengel) Balle (Loranthaceae) parasite of agroecosystems' fruit trees in Cameroon. Journal of Agricultural Biotechnology and Sustainable Development, 2(5): 076-081.

Din N, Seanger P, Priso JR, Dibong SD, Blasco F. 2008. Logging activities in mangrove forests: A case study of Douala Cameroon. African Journal of Environmental Science and Technology, 2(2): 22-30.

Engone Obiang NL, Sallé G. 2006. Faut-il éradiquer Phragmanthera capitata, parasite des hévéas en Afrique ? Comptes Rendus de Biologies, 329: 185-195.

Glatzel G. 1983. Mineral nutrition and water relations of hemiparasitic Mistletoe, Amyema preissii and its hosts, Acacia acuminate. Annales Botaniques, 49: 193201.

Levitt J. 1980. Responses of Plants to Environmental Stresses (United Kingdom edn). Edition Academic Press: London; 395-434.

Mony R, Ondoua JM, Dibong SD, Boussim IJ, Amougou A. 2009. Myrmécofaune arboricole associée aux couples Phragmanthera capitata (Sprengel) Balle/hôte au verger de la chefferie de 
Ndogbong (Douala, Cameroun). International Journal of Biological and Chemical Sciences, 3(6): 1346-1356.

Mony R, Ondoua JM, Dibong SD, Boussim IJ, Amougou A. 2010. Ants and Phragmanthera capitata (Sprengel) Balle (Loranthaceae) impacts on considerable damages caused on fruit trees of the Ndogbong (douala, Cameroun) chieftaincy's orchard. Journal of Agricultural Extension and Rural Development, 2(3): 048-053.

Munns R. 2002. Comparative physiology of salt and water stress. Plant Cell and Environment, 25: 239-250.

Norton DA, Carpenter MA. 1998. Mistletoes as parasites; host specificity and speciation. Trends in Ecology and Evolution, 13: 101-105.

Polhill R, Wiens D. 1998. Mistletoes of Africa. The Royal Botanic Gardens: Kew; p. 370 .
Shaw MR. 1994. Parasitoid host ranges. Parasitoid Community Ecology, BA Hawkins, W Sheehan (eds). Oxford University Press: New Yok; 111-144.

Sonké B, Kenfack D, Tindo M. 2000. Parasitisme de l'avocatier (Persea americana, Lauraceae) par les Loranthacées dans la région de Yaoundé (Cameroun). Fruits, 55: 325-331.

Taffouo VD. 2005. Variation de la réponse au stress salin chez cinq espèces de Légumineuses: étude des marqueurs physiologiques et biochimiques. Thèse d'Etat ès Sciences, Université de Yaoundé I, p. 150.

Thompson JN. 1994. The Coevolutionary Process. University of Chicago Press: Chicago.

Whittington, Sinclair R. 1988. Water relations of the Mistletoes, Amyema miquelli and its hosts Eucalyptus fasciculosa. Australia Journal of Botony, 36: 239-255. 